Supporting Information

\title{
Building Dynamic Cellular Machineries in Droplet-based Artificial Cells with Single-Droplet Tracking and Analysis
}

\author{
Meng Sun, Zhengda Li, Shiyuan Wang, Gembu Maryu, and Qiong Yang* \\ Department of Biophysics, University of Michigan, Ann Arbor, Michigan 48109, United States
}

\section{Table of Contents}

\begin{tabular}{|l|l|}
\hline Experimental setup w/Figure S1 & $\mathrm{S}-2$ \\
\hline Droplet analysis w/Figure S2 & $\mathrm{S}-2 \sim \mathrm{S}-3$ \\
\hline Securin-CFP plasmid map & $\mathrm{S}-3$ \\
\hline Experimental controls w/Figure S3 & $\mathrm{S}-3 \sim \mathrm{S}-4$ \\
\hline Modeling on transcription and translation dynamics w/Figures S4, S5, and Tables S1 & $\mathrm{S}-4 \sim \mathrm{S}-7$ \\
\hline Securin-CFP oscillations in droplets w/Figure S6 & $\mathrm{S}-7$ \\
\hline Discriminating mitotic and interphase droplet w/Figure S7 & $\mathrm{S}-7 \sim \mathrm{S}-8$ \\
\hline Mitotic and interphase duration simulation w/Tables S2 & $\mathrm{S}-8$ \\
\hline Movies S1, S2 & $\mathrm{S}-9$ \\
\hline References & $\mathrm{S}-9$ \\
\hline
\end{tabular}




\section{Experimental setup}

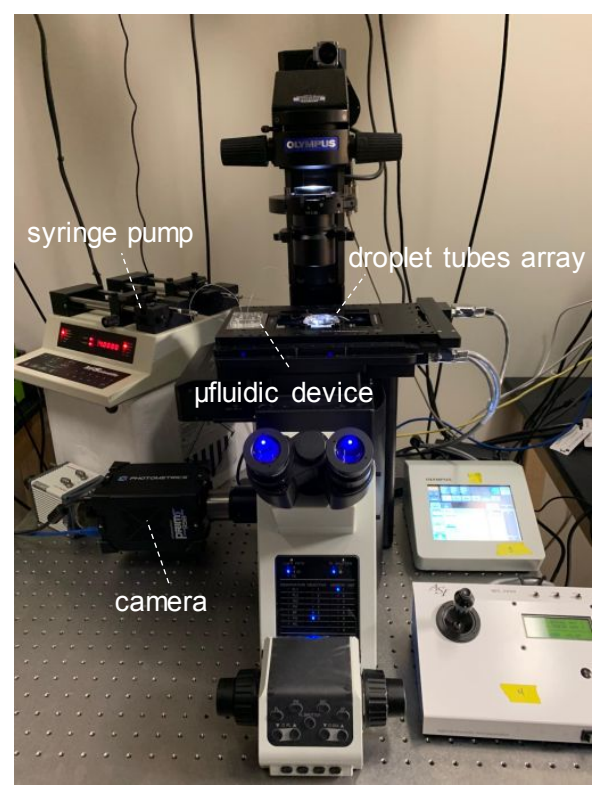

Figure S1. Experimental setup on an inverted microscope showing a syringe pump and a microfluidic device for droplets generation, an oil petri dish for droplets incubation, and an attached camera for droplets imaging.

\section{Droplet analysis}

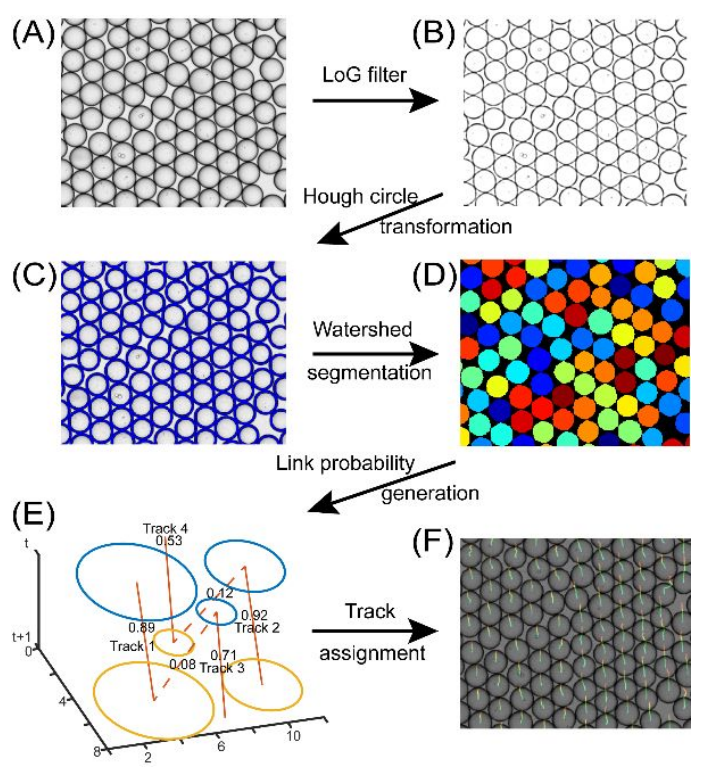

Figure S2. Image processing pipeline. (A) A section of an original Image. (B) Image after LoG filter. (C) Hough circle transformation followed by thresholding and overlap detection. (D) Watershed segmentation with seeds generated from circle detection. (E) Illustration of the linking process. 6 possible links found between two frames with 3 segments. The number next to tracks represent linking probability. After optimizing maximum probability, 4 of the links (solid line) are adopted and added to tracks, while the rest (dashed line) are discarded. Note that track 4 represents the droplet moving in from outside while track 3 represents the droplet moving out of the view (F) Example of tracking results.

Images are preprocessed with background subtraction to compensate illumination difference. An LoG (Laplacian of Gaussian) filter is then applied to the image to increase the contrast of edge and suppress noise. Hough circle transform is used to detect a circular object, and the detected circles are trimmed to avoid overlapping. Automated lineage tracking is performed on features calculated during segmentation. Segments are organized in K-D trees for faster searching. Droplets 
from each time point $T$ were compared with the ones from $T+1$ to search for the globally optimal matches between neighboring time points. ${ }^{[1]}$ Parameters used for tracking were automatically calculated based on the distribution of droplets features and confirmed in subsamples by eye. As an output from tracking, each track starts at first time point, or droplets move into the image field, and ends with last time point, or droplets move out of image field. After tracking, the individual droplet tracks are further filtered based on tracking length, droplet shape/size.

\section{Securin-CFP plasmid map and sequence}

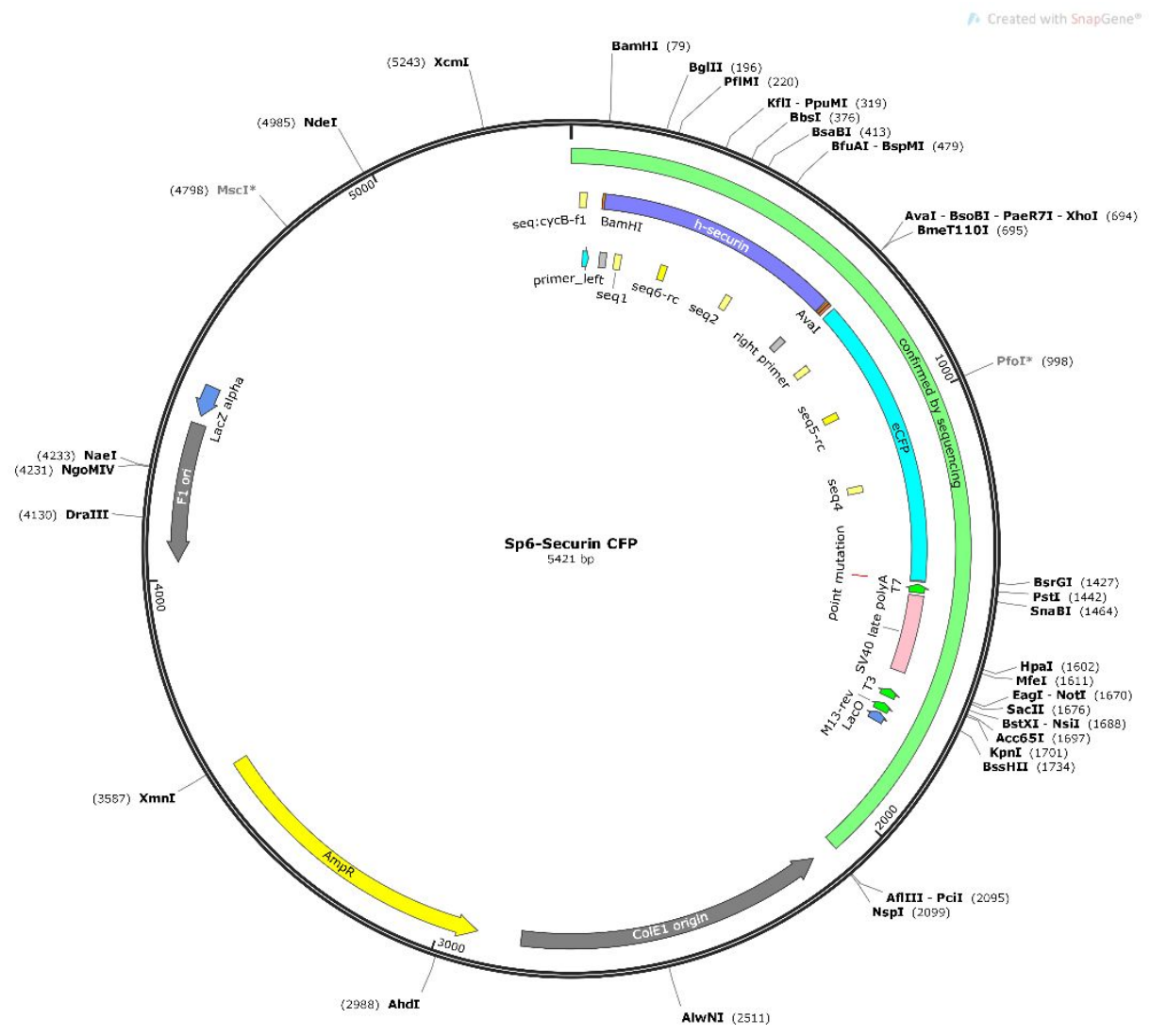

ORF sequence for Securin-flexible linker-CFP-stop codon:

atggctactctgatctatgttgataaggaaaatggagaaccaggcacccgtgtggttgctaaggatggg gctgaagctggggtctggaccttcaatcaaagccttagatgggagat ctcaagtttcaacaccacgttttggcaaaacgttcgatgccccaccagccttacctaaagctactagaaaggctttgggaactgtcaacagagctacagaaaagtctgtaaaga ccaagggacccctcaaacaaaaacagccaagcttttctgccaaaaagatgactgagaagactgttaaagcaaaaagctctgttcctgcctcagatgatgcctatccagaaat agaaaaattctttcccttcaatcctctagactttgagagttttgacctgcctgaagagcaccagattgcg cacctccccttgagtggagtgcctctcatgatccttgacgaggagaga gagcttgaaaagctgtttcagctgggccccccttcacctgtgaagatg ccctctccaccatgg gaatccaatctgttg cagtctccttcaag cattctgtcgaccctg gatgttgaatt gccacctgtttgctgtgacatagatattGGTCTCGAGCCTCTAGAAGGTGGCGGAatggtgagcaagggegaggagctgttcaccggggtggtgcccatcct ggtcgagctggacggcgacgtaaacggccacaagttcagcgtgtccggcgagggcgagggcgatgccacctacggcaagctgaccctgaagttcatctgcaccaccggc aagctgcccgtgccetggcccaccctcgtgaccaccctgacctggggcgtgcagtgcttcagccgctaccccgaccacatgaagcagcacgacttcttcaagtccgccatgcc cgaaggctacgtccaggagcgcaccatcttcttcaaggacgacggcaactacaagacccgcgccgaggtgaagttcgagggcgacaccctggtgaaccgcatcgagctg aagggcatcgacttcaaggaggacggcaacatcctggggcacaagctggagtacaactacatcagccacaacgtctatatcaccgccgacaagcagaagaacggcatca aggccaacttcaagatccgccacaacatcgaggacggcagcgtgcagctcgccgaccactaccagcagaacacccccatcggcgacggccccgtgctgctgcccgaca accactacctgagcacccagtccgccctgagcaaagaccccaacgagaagcgcgatcacatggtcctgctggagttcgtgaccgccgccgggatcactctcggcatggacg agctgtacaagTAA

\section{Experimental controls}

We have performed the TXTL and TL experiments in a conventional microplate reader with non-securin DNA (CFP Plasmid in Extract) and non-securin mRNA (CyclinB-CFP mRNA in Extract) as positive controls, and lysate-only (Extract) and 
plasmid-only (Securin-CFP Plasmid) as negative controls. Each reaction has been duplicated. The positive controls indicate that the assays were not limited to the synthesis of securin, any mRNA or genes with SP6 promoter can also be expressed in the SP6 Wheat Germ extract. The negative controls prove that both the lysate and plasmid background signals remained stably low over the entire assay, and that these background signals unlikely cause any artifact to the real signals in the Securin-CFP TXTL and TL experiments. The surfactant oil did not affect the assay either. We did find that the variations for all the plasmid samples were significantly higher than the mRNA samples, which is consistent with our droplet results shown in Figure 3 of the main text. However, limited by the large volume of cell extract consumed by the microplate assays (20,000 times more than droplet assay), we only managed to generate three repeats of these experiments on three different days. The three repeats of experiments yielded similar results (data on other days are not shown). Currently, we don't fully understand the source of this variation in the microplate experiments. The possible explanations for the larger variations in TXTL experiments compared with TL experiments could include stochastic gene expression (e.g. transcription bursts), the scarcity of transcriptional machinery, and pipetting errors and other experimental handling errors, but fully investigating this question is beyond the scope of this paper.
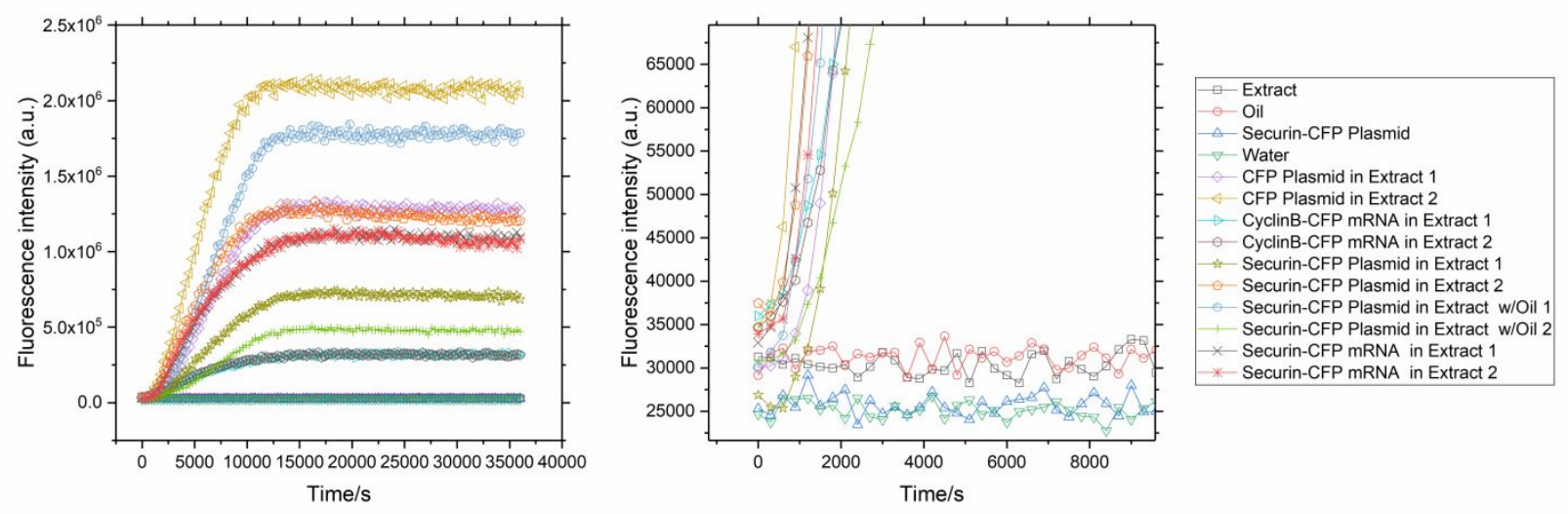

Figure S3. Control experiments on a conventional microplate reader. Samples were tested in duplicates. Reaction volume: $10 \mu \mathrm{L}$. Ex: $430 \mathrm{~nm}$, Em: $475 \mathrm{~nm}$. Temperature: $24^{\circ} \mathrm{C}$. Plasmid and mRNA added in total: CFP Plasmid $1.9 \mu \mathrm{g}$, CyclinB-CFP mRNA $3.0 \mu \mathrm{g}$, Securin-CFP Plasmid $1.9 \mu \mathrm{g}$, and Securin-CFP mRNA $1.8 \mu \mathrm{g}$. Duplicate measurements are shown for samples (securin-CFP plasmid and mRNA in extracts) and positive controls (CFP plasmid and Cyclin B-CFP mRNA in extracts). Only one representative curve for each of the negative controls (i.e. Extract, Oil, Securin-CFP plasmid only, water) is demonstrated in the plot. The figure on the right is a zoom-in of the figure on the left.

\section{Modeling on transcription and translation dynamics}
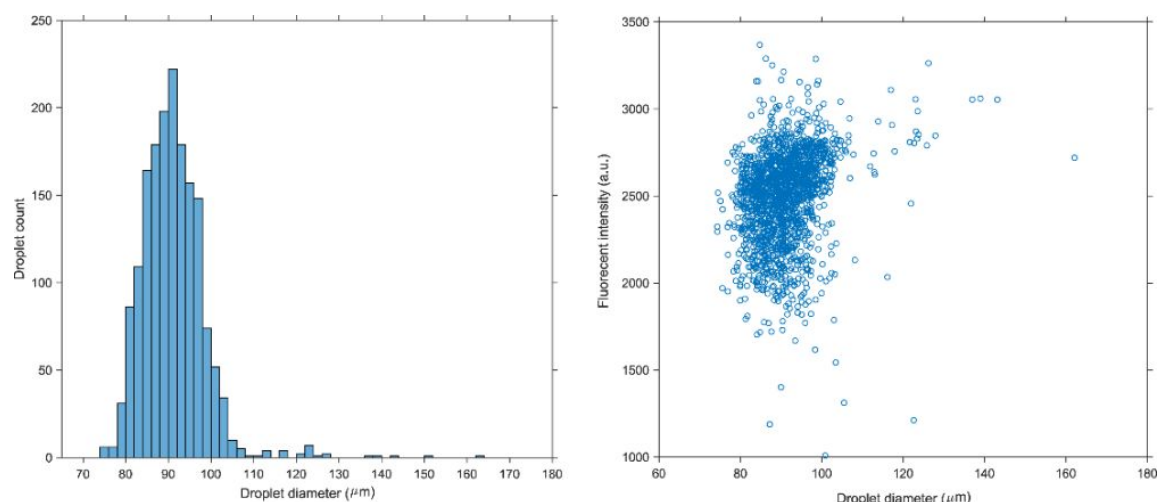

Figure S4. The fluorescence intensity and droplet size distributions at a frame of 600 min in Figure 3B. (A) Size distribution of droplets. (B) Droplet intensity is not correlated with droplet size, indicating that the droplet size is not an effector for the wider variation of the droplet intensity in the TXTL assay.

To fit experimental result, we utilized the model by Joachim O. Rädler's group ${ }^{[2]}$ for TXTL system (1) (5) and also modified it to simulate the TL system (6) (9). Here, [mRNA], [CFP], [CFP*], [TsR] and [TIR] are defined as securin-CFP mRNA concentration, primitive securin-CFP concentration, matured securin-CFP concentration, normalized transcription resource and normalized translation resource respectively. The resource is an abstract value representing all matters and energies participated in transcription and translation. 


$$
\begin{aligned}
& \frac{\mathrm{d}[m R N A]}{d t}=\frac{k_{t s} \times[T s R] \times[D N A]}{K_{s}+[D N A]}-\delta_{m R N A} \times[m R N A] \text { (1) } \\
& \frac{d[C F P]}{d t}=\frac{k_{t l} \times[T l R] \times[m R N A]}{K_{l}+[m R N A]}-k_{m a t} \times[C F P](2) \\
& \frac{d\left[C F P^{*}\right]}{d t}=k_{m a t} \times[C F P]^{(3)} \\
& \frac{d[T s R]}{d t}=-\frac{k_{c s} \times[T s R] \times[D N A]}{K_{s}+[D N A]}(4) \\
& \frac{d[T l R]}{d t}=-\frac{\delta_{T l R} \times[T l R]}{K_{T l R}+[T l R]}(5) \\
& \frac{\mathrm{d}[m R N A]}{d t}=-\delta_{m R N A} \times[m R N A] \\
& \frac{d[C F P]}{d t}=\frac{k_{t l}[T l R][m R N A]}{k_{l}+[m R N A]}-k_{m a t} \times[C F P](7) \\
& \frac{d\left[C F P^{*}\right]}{d t}=k_{\text {mat }} \times[C F P](8) \\
& \frac{d[T l R]}{d t}=-\frac{\delta_{T l R} \times[T l R]}{K_{T l R}+[T l R]} \text { (9) }
\end{aligned}
$$

In our system, the initial DNA concentration (80ng/ $\mu \mathrm{l})$ in the TXTL system and mRNA concentration $(74 \mathrm{ng} / \mu \mathrm{l})$ in TL system is high enough to assume that their concentration in each droplet is the same. Since DNA degradation is slow, $\frac{k_{t s}[D N A]}{K_{s}+[D N A]}$ can be simplified as one parameter $k_{t s}$. In addition, we assume that the observed fluorescence intensity is linearly correlated with the concentration of CFP, thus after baseline subtraction, we have $I=a[C F P]$ where $\mathrm{I}$ is the observed fluorescence intensity, and a is scaling factor. After nondimensionalization of all concentrations, the equations for the TXTL system (10) (14) and Tl-only system (15) (18) are shown below.

$$
\begin{aligned}
& \frac{\mathrm{d}\left[R^{\prime}\right]}{d t}=\left[T s R^{\prime}\right]-\delta_{m R N A} \times\left[R^{\prime}\right](10) \\
& \frac{d\left[C^{\prime}\right]}{d t}=\frac{\left[T l R^{\prime}\right] \times\left[R^{\prime}\right]}{1+\left[R^{\prime}\right]}-k_{m a t} \times\left[C^{\prime}\right](11) \\
& \frac{d\left[C^{*}\right]}{d t}=\left[C^{\prime}\right](12) \\
& \frac{d\left[T s R^{\prime}\right]}{d t}=-k_{c s}{ }^{\prime} \times\left[T S R^{\prime}\right](13) \\
& \frac{d\left[T l R^{\prime}\right]}{d t}=-\frac{\delta_{T l R}{ }^{\prime} \times\left[T l R^{\prime}\right]}{1+\left[T l R^{\prime}\right]}(14)
\end{aligned}
$$




$$
\begin{aligned}
& \frac{\mathrm{d}\left[R^{\prime}\right]}{d t}=-\delta_{m R N A} \times\left[R^{\prime}\right](15) \\
& \frac{d\left[C^{\prime}\right]}{d t}=\frac{\left[T l R^{\prime}\right] \times\left[R^{\prime}\right]}{1+\left[R^{\prime}\right]}-k_{m a t} \times\left[C^{\prime}\right](16) \\
& \frac{d\left[C^{*}\right]}{d t}=\left[C^{\prime}\right](17) \\
& \frac{d\left[T l R^{\prime}\right]}{d t}=-\frac{\delta_{T l R} \times\left[T l R^{\prime}\right]}{1+\left[T l R^{\prime}\right]}(18)
\end{aligned}
$$

Since both the TXTL and TL-only systems are constructed using the same wheat germ extract, we assume that the resource consumption parameter $\delta_{T l R}$, the maturation rate of CFP, $k_{\text {mat }}$, and the initial resources share the same values for the two systems. We fit the model to our experimental data with these constraints. The fitting is performed using a trust-region algorithm in MATLAB. We randomly selected 50 points around values from previously published work as initial conditions, ${ }^{[2]}$ and used the best fit (with the highest r-square values) to estimate the parameters. The fit parameter values are shown in Table S1 and the fit curves shown in Figures 3B and 3D. Note that due to nondimensionalization, initial [TIR] and [TsR] concentrations are also considered as fitting parameters. For comparison's purpose, we calculated the maturation rate of CFP protein to be $64 \mathrm{~min}$, slightly higher than that measured in yeast ( 50min) ${ }^{[3]}$

Table S1. Parameter fitting for transcription-translation model

\begin{tabular}{lll}
\hline & TI only & TxTI \\
\hline $\mathrm{k}_{\text {mat }}$ & $0.0157 \mathrm{~min}^{-1}$ & $0.0157 \mathrm{~min}^{-1}$ \\
\hline$\delta_{\text {ma }}$ & $0.0072 \mathrm{~min}^{-1}$ & $6.6 \mathrm{e}-6 \mathrm{~min}^{-1}$ \\
\hline$\delta_{\mathrm{tr}}{ }^{\prime}$ & $0.07 \mathrm{~min}^{-1}$ & $0.07 \mathrm{~min}^{-1}$ \\
\hline $\mathrm{a}$ & 0.015 & 1.269 \\
\hline $\mathrm{k}_{\mathrm{cs}}{ }^{\prime}$ & & $2 \mathrm{e}-6 \mathrm{~min}^{-1}$ \\
\hline Initial [TIR] & 23.7 & 23.7 \\
\hline Initial [TsR] & & $3.95 \mathrm{e}-5$ \\
\hline
\end{tabular}

The fluorescence intensity time series of individual droplets in the TXTL system have a larger variation than the TL-only system, which would be difficult to observe in bulk measurement. The standard deviations of their fluorescence intensities are plotted in Figure 3E. Initially, the standard deviations of both systems are rather similar, but the difference is amplified starting around $200 \mathrm{~min}$. We assume that the difference may stem from the initial non-uniform distributions of molecules, and the additional transcription step in the TXTL system can further amplify them. In our study, the initial number of DNA or mRNA molecules in each droplet is high enough and is unlikely to contribute to the large variations among the droplets, but other transcription and/or translation factors may contribute to this process. ${ }^{[4]}$ To verify this hypothesis, we fit each individual time series using our model with all parameters fixed at the best-fit values in Table S1 except that the initial transcription resources and initial translation resources are freely changing parameters. Surprisingly, with only one variable in the TL-only system and two variables in the TXTL-coupled system, the model reliably fit the time series for all droplets. The adjusted $r$ squared values for the TL-only and TXTL systems are shown in Figures S5A and S5B. The results suggest that the variation between droplets can be explained by initial resource difference alone. In the TXTL-coupled system, we observed a negative correlation between the translation resource and transcription resource, which can be explained by limited resources like energy shared by both processes. The relative log deviation from the median is shown in Figure S5D, suggesting the variations of initial translation resources are similar in the TXTL and TL systems. The result also shows that the initial transcription resource has a larger variation than that of translation, indicating that the transcription machinery in extract might be scarcer and more sensitive to the diversity caused by microdroplet encapsulation. 
(A)

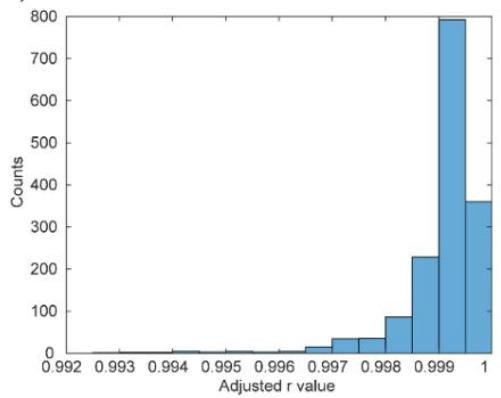

(C)

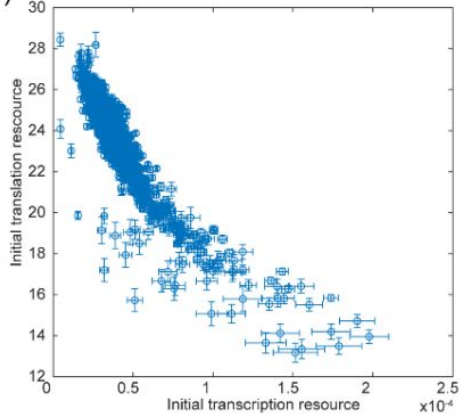

(B)

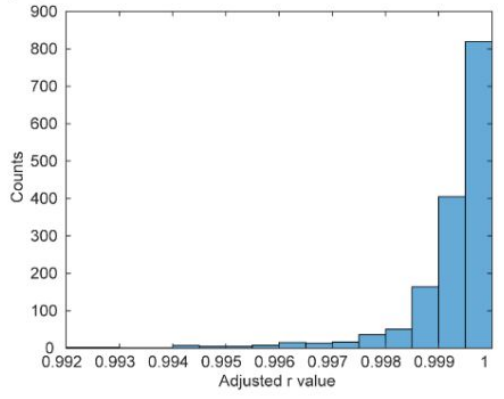

(D)

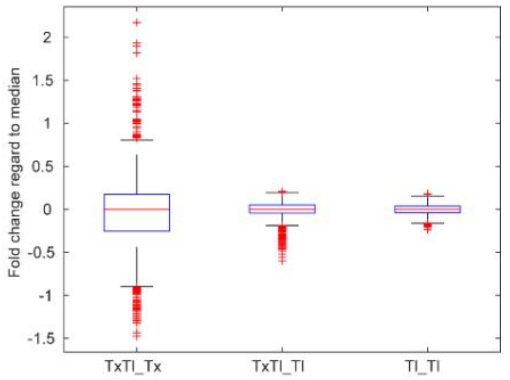

Figure S5. Fitting the dynamics of TXTL system and TL system. (A) Distribution of adjusted r-square for the fitting of each individual curve in translation only system. (B) Distribution of adjusted r-square for fitting in transcription-translation coupled system. (C) Relationship between the fitted value of the initial transcription resource and initial translation resource. Each point is unique fitting on a time series. Error bar indicates $95 \%$ confidence interval of the parameter values during fitting. (D) Comparison of parameter variation. Showing the distribution of log2(parameters/median of parameters). From left to right, transcription resource in TXTL system, translation resource in TXTL system, translation resource in TL-only system.

\section{Securin-CFP oscillations in droplets}

(A)

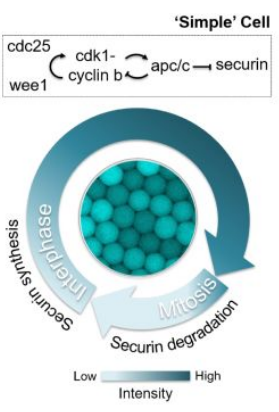

(B)

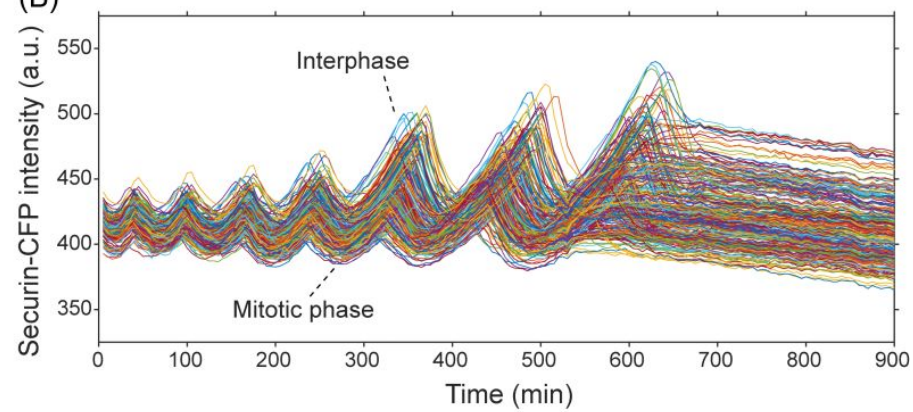

Figure S6. (A) A 'simple' cell oscillator drives alternations of synthesis and degradation of securing-CFP. Top scheme indicates the circuit of a 'simple' cell. The inner image shows a portion of the fluorescent image of the droplet oscillators from the data set of (B). (B) Fluorescence intensities of securinCFP indicating securin synthesis and degradation in cell cycles of 376 droplet oscillators.

\section{Discriminating mitotic phase and interphase in droplets}

In cell cycle reconstruction assay, one of the major challenges for cell cycle period estimation is to accurately determine cell cycle states. Here we used the nuclear envelope as an indicator. When there is an intact nucleus, the droplets are assigned to be interphase, otherwise mitotic phase. In this research, for simplicity, we used the standard variation of fluorescent intensity in a droplet as a measurement for nucleus appearance. However, we observed that the exact threshold of standard deviation that could be classified as the value changes with background intensity. To reliably classify each time point, we manually labeled the states of 1000 droplets and use its average and standard deviation of fluorescent intensity as features for supervised learning. We used a Support Vector Machine (SVM) as a classifier, the result of classification is shown in Figure S7. 

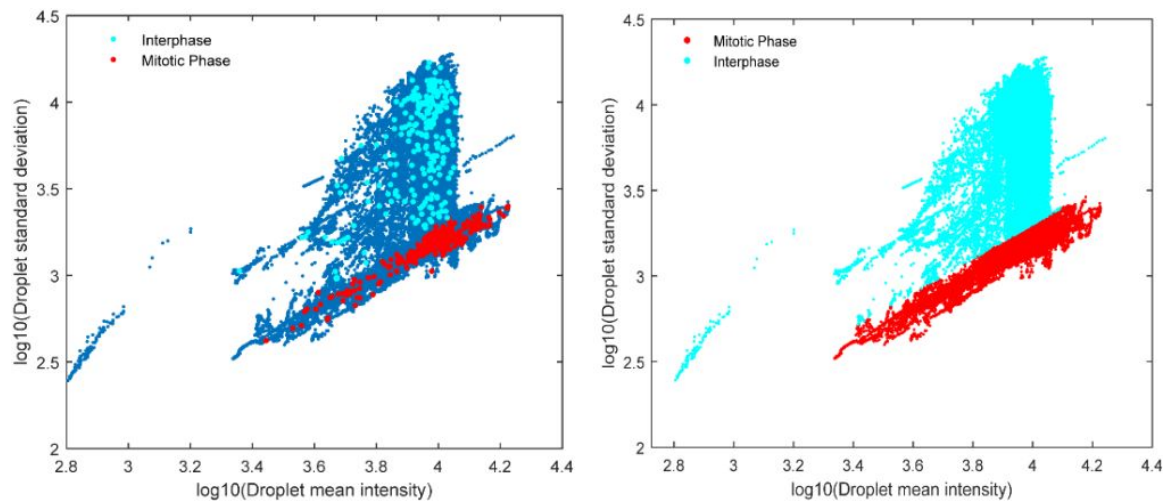

Figure S7. Supervised learning to discriminate interphase and mitotic phase droplets. The scatter plot shows the relationship between droplet mean intensity and droplet intensity standard variation. (A) A training set of mitotic phase (red) and interphase (cyan) is manually labeled by an expert, on top of all raw data points (blue). (B) Classification generated by support vector machine, that separates the mitotic phase (red) from interphase (cyan).

\section{Mitotic and interphase duration simulation}

We used a well-established model to simulate the cell cycle in Xenopus extract.[5,6]

$$
\begin{aligned}
\frac{d}{d t}[C y c \operatorname{lin} B] & \\
& =k_{s y}-k_{\text {deg }}[C y \operatorname{clin} B]=k_{s y}-\left(a_{\text {deg }}+\frac{b_{\text {deg }}\left[C d k 1_{a}\right]^{n_{\text {deg }}}}{\left[C d k 1_{a}\right]^{n_{\text {deg }}}+E C 50_{\text {deg }}^{n_{\text {deg }}}}\right)[C y c l i n B]
\end{aligned}
$$

Eq.1

$$
\frac{d}{d t}\left[C d k 1_{a}\right]
$$

$$
\begin{gathered}
=k_{s y}+\frac{1}{\sqrt{r}}\left(a_{C d c 25}+\frac{b_{C d c 25}\left[C d k 1_{a}\right]^{n_{C d c 25}}}{\left[C d k 1_{a}\right]^{n_{C d c 25}}+E C 50_{C d c 25}^{n_{C d 25}}}\right)\left([C y c l i n B]-\left[C d k 1_{a}\right]\right)-\sqrt{r} \\
\left(a_{W e e 1}+\frac{b_{W e e 1} E C 50_{W e e 1}^{n_{W e e 1}}}{\left[C d k 1_{a}\right]_{\text {Wee1 }}^{n_{\text {Wee }}}+E C 50_{\text {Wee } 1}^{n_{\text {Wee }}}}\right)\left[C d k 1_{a}\right]-\left(a_{d e g}+\frac{b_{d e g}\left[C d k 1_{a}\right]^{n_{\text {deg }}}}{\left[C d k 1_{a}\right]^{n_{\text {deg }}}+E C 50_{\text {deg }}^{n_{\text {deg }}}}\right)\left[C d k 1_{a}\right] \\
E q .2
\end{gathered}
$$

The parameters for the model are listed below:

Table S2. Parameters of cell cycle oscillation model

\begin{tabular}{ll}
\hline ksy & $1 \mathrm{nM} / \mathrm{min}$ \\
\hline awee1 & $0.08 \mathrm{nM} / \mathrm{min}$ \\
\hline bwee1 & $0.4 \mathrm{nM} / \mathrm{min}$ \\
\hline nwee1 & 3.5 \\
\hline EC50wee1 & $35 \mathrm{nM}$ \\
\hline acdc25 & $0.16 \mathrm{nM} / \mathrm{min}$ \\
\hline bcdc25 & $0.8 \mathrm{nM} / \mathrm{min}$ \\
\hline ncdc25 & 11 \\
\hline EC50cdc25 & $30 \mathrm{nM}$ \\
\hline adeg & $0.01 \mathrm{nM} / \mathrm{min}$ \\
\hline bdeg & $0.04 \mathrm{nM} / \mathrm{min}$ \\
\hline ndeg & 17 \\
\hline EC50deg & $32 \mathrm{nM}$ \\
\hline
\end{tabular}


The cell cycle state in the model is determined by the concentration of CDK1-cyclinB molecules when the active CDK1 level is higher than a threshold (here the threshold is set to be 15000), the droplet is considered to be at mitotic phase. Due to the steep increase and decrease of CDK1 activity curve, the selection of threshold does not have a significant impact on the relative duration of interphase and mitotic phase.

\section{Movies}

Movie S1. Droplet indexing and tracking in a 5-h period. More mobile droplets in a relatively loose pack could also be successfully tracked over extended periods (movie not shown).

Movie S2. Nuclear envelop formation and breakdown during cell cycle oscillations in droplets.

\section{References}

(1) S. Huh, D. F. E. Ker, R. Bise, M. Chen, T. Kanade, IEEE Trans. Med. Imaging, 2011, 30, 586-596.

(2) T. Stogbauer, L. Windhager, R. Zimmer, J. O. Radler, Integr. Biol. 2012, 4, 494-501.

(3) A. Gordon, A. Colman-Lerner, T. E. Chin, K. R. Benjamin, R. C. Yu, R. Brent, Nat Methods 2007, 4, 175-181.

(4) K. Kapsner, F. Simmel, ACS Synth. Biol. 2015, 4, 1136-1143.

(5) Y. Guan, Z. Li, S. Wang, P. M. Barnes, X. Liu, H. Xu, M. Jin, A. P. Liu, Q. Yang, eLife 2018, 7, e33549.

(6) Q. Yang, J. E. Ferrell Jr. Nat. Cell Biol. 2013, 15, 519-525. 
S-10 